\title{
A case of acute hemorrhagic edema of infancy
}

\section{Aastha Wadhwa, Anjan Kumar Patra, Bijina Kolukulangara Dharman}

\author{
Department of Dermatology, MVJ Medical College and Research Hospital, Bangalore, India
}

Corresponding author: Dr. Aastha Wadhwa, E-mail: dr.aastha01@gmail.com

\begin{abstract}
Acute hemorrhagic edema of infancy (AHEI) is a cutaneous leukocytoclastic vasculitis of infants, clinically characterized by acute development of peripheral edema and targetoid purpuric lesions on face and extremities. It is considered to be an uncommon form of cutaneous vasculitis occurring in children younger than two years old. The clinical picture has a violent onset, a short benign course followed by spontaneous complete recovery. We report a case of a male child who presented with upper respiratory tract infection followed by limb swelling and purpuric and ecchymotic lesions on the skin. Skin biopsy revealed leukocytoclastic vasculitis.
\end{abstract}

Key words: Acute hemorrhagic edema of infancy; Ecchymoses; Leukocytoclastic vasculitis; Purpura

\section{INTRODUCTION}

Acute hemorrhagic edema of infancy (AHEI) is a form of benign cutaneous leukocyteclastic vasculitis [1]. It affects children younger than two years of age. Respiratory tract infections, immunization, and drug intake are considered as precipitating factors. AHEI is characterized by fever, ecchymotic,purpuric lesions, and edema on the extremities and/or face. On histopathology, the skin lesions show leukocytoclastic vasculitis. The clinical features may be confused with Henoch-Schönlein purpura, erythema multiforme, meningococcemia, and septicemia. There is no internal organ involvement, and the disease runs a benign course with spontaneous resolution [2].

We Report a case of AHEI in a one year old male child.

\section{CASE REPORT}

A one year old male child presented to our opd with complaint of red raised Itchy lesions over lower limbs, buttocks, right arm and ear pinna since one day (Figs. 1 and 2). He had symptoms of upper respiratory tract infection, swelling of lower limb, initially started over the right foot and gradually progressed to involve the left foot and both legs till knee, and fever one episode and vomiting four days prior to the eruption of the skin lesions. He had no complaints of diarrhea, joint pain, or abdominal pain. On examination, the child was active and playful with stable vitals. General examination revealed bilateral inguinal lymphadenopathy and grade two non-pitting edema. The cutaneous examination showed multiple symmetrically distributed, round and oval, edematous, ecchymotic, purpuric, targetoid plaques localized on the both feet, legs, thighs, buttocks and pinna of right ear. Erythematous targetoid macules were present on the soles. There was no mucosal involvement. Systemic Examination was normal. Laboratory investigations, including hemoglobin, complete blood count, liver and renal function tests, and stool and urine examination, were normal. Skin Biopsy showed features of leucocytoclastic Vasculitis and Dermal edema (Fig. 3). DIF showed perivascular C3 deposits. He was treated with short course of oral steroids and supportive therapy there was complete resolution of the skin lesions within one week (Fig. 4).

\section{DISCUSSION}

Acute Hemorrhagic edema of infancy was first described by Snow in 1913 [3]. It is an acute Leukocytoclastic vasculitis occurring in infants and young children between the ages of three months and two years.

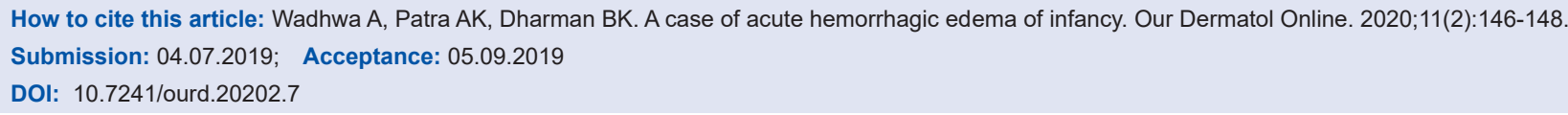




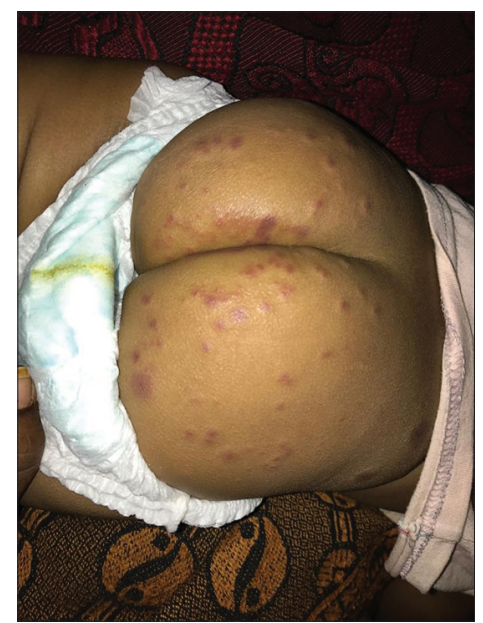

Figure 1: Erythematous papules with few targetoid lesions on buttocks.

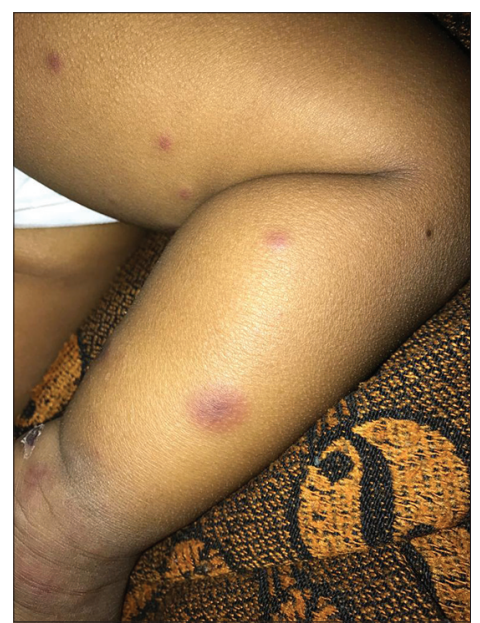

Figure 2: Erythematous edematous targetoid lesions present on the lower limbs.

Since its first description in 1913, approximately 100 cases have been reported [4]. It is also known as Finkelstein disease or medallion-like purpura or infantile postinfectious iris-like purpura and edema [5].

It is characterized by a triad of fever, large palpable purpuric skin lesions, and edema [6].

Acute hemorrhagic edema of infancy is an immune complex mediated vasculitis, which is probably initiated from prior bacterial or viral infections (mostly upper respiratory tract and urinary infection) in addition to some medications especially antibiotics and with less probability, vaccination. The most reported infective agents include Staphylococci, Streptococci and among viruses, Adenovirus, although many other agents, such as Escherichia coli, or Mycobacteria, have been reported [4]. Males are more susceptible than females. Painless non-pitting

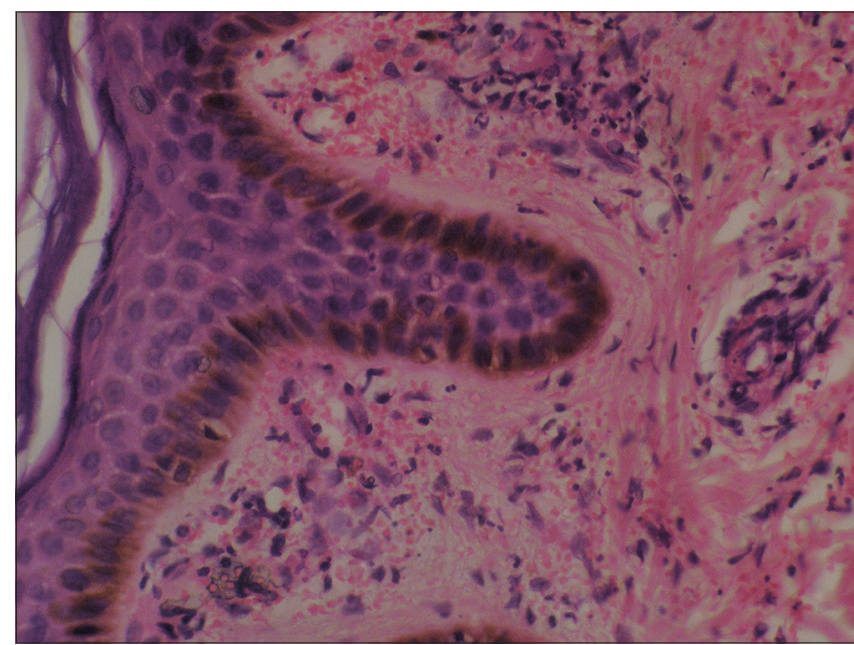

Figure 3: Histopathology showing leucocytoclastic vasculitis with extravasation of RBC's and Fibrinoid necrosis of vessel wall.

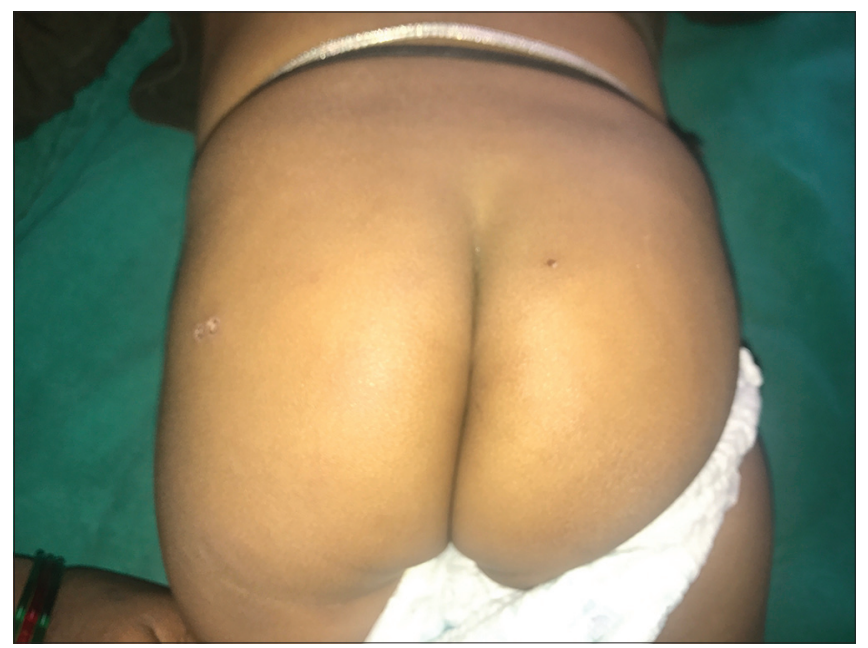

Figure 4: Response seen with short course of oral steroids and supportive measures within a week.

edema of the face, mostly asymmetric, may be the first clinical sign. Later on, painful ecchymotic patches and plaques appear on the face and extremities or large target shaped lesions may erupt suddenly. In contrast to striking cutaneous lesions and rapid progression, the overall general condition is good and babies are nontoxic [7]. Visceral involvement is rare but it has been reported involving the kidneys and intestines, causing symptoms such as hematuria, mild proteinuria, and bloody diarrhea [8]. Laboratory investigations are usually nonspecific. Most of the affected patients have a normal erythrocyte sedimentation rate and C-reactive protein level. Slight increase in the leukocytic count with lymphocytic line shift, neutrophilia or eosinophilia can be seen. The coagulation profile is almost always normal. Urinalysis and stool sample are often negative for blood. ASO 
titer, antinuclear antibodies, antideoxyribonucleic acid, and rheumatoid factor are usually negative. High serum levels of gamma globulins and immune complexes have been described [9]. Histologic features of AHEI are consistent with small vessel vasculitis of both capillaries and post capillary venules of the upper and the middle dermis, showing typical leukocytoclastic vasculitis with or without fibrinoid necrosis and a deep perivascular and interstitial infiltrate composed mostly of neutrophils with abundant nuclear dust [4]. The chief differential diagnosis of AHEI is Henoch schonlein purpura. The age of onset of Acute haemorrhagic edema of infancy is two to twenty four months is lower when compared to Henoch schonlein purpura four to seven years. Systemic complications (arthralgia, gastrointestinal bleeding, and nephritis) are common in Henoch schonlein purpura. The disease runs a benign course with complete spontaneous recovery occurring in one to three weeks, although relapses have been reported rarely. Treatment with oral corticosteroids has been reported, but this is unnecessary due to its spontaneous recovery [10].

\section{CONCLUSION}

Acute haemorrhagic edema of infancy is a benign disorder despite its dramatic appearance There is a contrast between the acuteness of skin lesions and good general condition of the patient. It is an unique disorder and needs to be differentiated from Henoch schonlein purpura.

\section{Consent}

The examination of the patient was conducted according to the Declaration of Helsinki principles.

\section{REFERENCES}

1. Taieb A, Legrain V. Acute hemorrhagic edema of the skin in infancy. In: Textbook of pediatric Dermatology ( $2^{\text {nd }}$ ed). United Kingdom. Blackwell Science; 2002:1569-73.

2. Dongre A, Adhe V, Kothari D, Kardekar S, Khopkar U. Acute hemorrhagic edema of infancy: A report of two cases. Indian J Dermatol Venereol Leprol. 2012;78:121.

3. Snow IM. Purpura, urticaria, and angioneurotic edema of the hands and feet in a nursing baby. JAMA. 1913;61:18-9.

4. Saraclar Y, Tinaztepe K, Adalioglu G, Tuncer A. Acute hemorrhagic edema of infancy (AHEI) - A variant of Henoch-Schönlein purpura or a distinct clinical entity? J Allergy Clin Immunol. 1990;86:473-83.

5. Shah D, Goraya JS, Poddar B, Parmar VR. Acute infantile hemorrhagic edema and Henoch-Schönlein purpura overlap in a child. Pediatr Dermatol. 2002;19:92-3.

6. McDougall CM, Ismail SK, Osmerod A. Acute hemorrhagic oedema of infancy. Arch Dis Child. 2005;90:316.

7. da Silva Manzoni AP, Viecili JB, de Andrade CB, Kruse RL, Bakos L, et al. Acute hemorrhagic edema of infancy: A case report. Int J Dermatol. 2004;43:48-51.

8. Garty BZ, Ofer I, Finkelstein Y. Acute hemorrhagic edema of infancy. Isr Med Assoc J. 2002;4:228-9.

9. Alharbi MS. Role of systemic steroids in acute hemorrhagic edema of infancy: report of two cases. Int J Adv Med. 2019;6:537-41.

10. Jindal SR, Kura MM. Acute hemorrhagic edema of infancy-a rare entity. Indian Dermatol Online J. 2013;4:106-8.

Copyright by Aastha Wadhwa, et al. This is an open-access article distributed under the terms of the Creative Commons Attribution License, which permits unrestricted use, distribution, and reproduction in any medium, provided the original author and source are credited.

Source of Support: Nil, Conflict of Interest: None declared. 